JOURNAL OF

APPLIED

CRYSTALLOGRAPHY

ISSN 1600-5767

Received 13 October 2017

Accepted 22 March 2018

Edited by M. Arai, European Spallation Source, Lund, Sweden

${ }^{1}$ This article will form part of a virtual special issue on advanced neutron scattering instrumentation, marking the 50th anniversary of the journal.

Keywords: neutron diffraction; stroboscopic experiments; event-based data processing; spectroscopy.

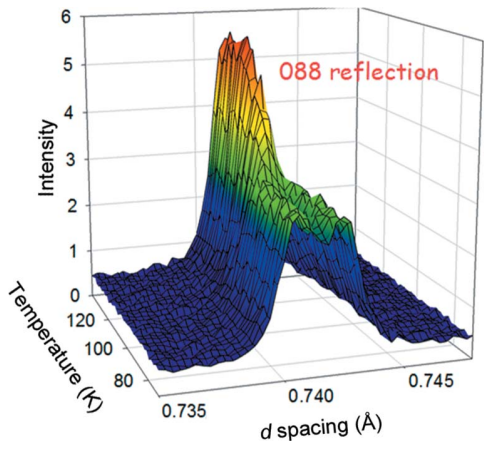

\section{Event-based processing of neutron scattering data at the Spallation Neutron Source ${ }^{1}$}

\author{
Garrett E. Granroth, ${ }^{\mathrm{a} *}$ Ke An, ${ }^{\mathrm{a}}$ Hillary L. Smith, ${ }^{\mathrm{b}}$ Pamela Whitfield, ${ }^{\mathrm{a}, \mathrm{c}}$ Joerg C. \\ Neuefeind, ${ }^{a}$ Jooseop Lee, ${ }^{\text {a,d }}$ Wenduo Zhou, ${ }^{a}$ Vladislav N. Sedov, ${ }^{\text {e }}$ Peter F. \\ Peterson, ${ }^{a}$ Andre Parizzi, ${ }^{a}$ Harley Skorpenske, ${ }^{a}$ Steven M. Hartman, ${ }^{\text {A Ashfia Huq }}{ }^{a}$ \\ and Douglas L. Abernathy ${ }^{\text {a }}$
}

${ }^{\mathbf{a}}$ Neutron Scattering Division, Oak Ridge National Laboratory, Oak Ridge, Tennessee, USA, ${ }^{\mathbf{b}}$ California Institute of Technology, Department of Applied Physics and Materials Science, Pasadena, California, USA, ${ }^{\mathbf{P}}$ Paul Scherrer Institute, 5232 Villigen PSI, Switzerland, ' $\mathrm{CHESS}$, Cornell University, Ithaca, New York, USA, and ${ }^{\mathbf{e}}$ Neutron Technology Division, Oak Ridge National Laboratory, Oak Ridge, Tennessee, USA. *Correspondence e-mail: granrothge@ornl.gov

The Spallation Neutron Source at Oak Ridge National Laboratory, USA, ushered in a new era of neutron scattering experiments through the use of eventbased data. Tagging each neutron event allows pump-probe experiments, measurements with a parameter asynchronous to the source, measurements with continuously varying parameters and novel ways of testing instrument components. This contribution will focus on a few examples. A pulsed magnet has been used to study diffraction under extreme fields. Continuous ramping of temperature is becoming standard on the POWGEN diffractometer. Battery degradation and phase transformations under heat and stress are often studied on the VULCAN diffractometer. Supercooled $\mathrm{Al}_{2} \mathrm{O}_{3}$ was studied on NOMAD. A study of a metallic glass through its glass transition was performed on the ARCS spectrometer, and the effect of source variation on chopper stability was studied for the SEQUOIA spectrometer. Besides a summary of these examples, an overview is provided of the hardware and software advances to enable these and many other event-based measurements.

\section{Introduction}

One of the novel features of the Spallation Neutron Source (SNS) (Mason et al., 2006) at Oak Ridge National Laboratory is event-based data acquisition. At pulsed sources, the wavelength (or energy) of the neutron is determined by a time-offlight-based measurement of the neutron velocity. Therefore, diffraction is measured using a wavelength-resolved Laue technique, as illustrated in Fig. 1 (Windsor, 1981). For the previous generation of spallation sources, the data-acquisition electronics have saved data as a series of bins in time of flight and position. As neutrons from each pulse scatter from the sample, they are added to the histogram for each bin. This method was well matched to the power of these sources and the speed of their acquisition electronics. Some experiments that produced sub-histograms have been performed at ISIS (Rutherford Appleton Laboratory, Oxfordshire, UK) to reach millisecond time scales (Steigenberger et al., 1994, 1995; Dalgliesh et al., 2004). In contrast, the new generation of spallation sources, both the SNS and J-PARC (Tokai, Ibaraki, Japan; Satoh et al., 2009; Nakatani et al., 2014), use eventbased recording. Here, each neutron event is recorded as a pixel position and a time stamp. So now time-dependent processes that occur as fast as the data-acquisition clock and stroboscopic experiments are straightforward to implement. Similar event-based collection has been used in the high- 


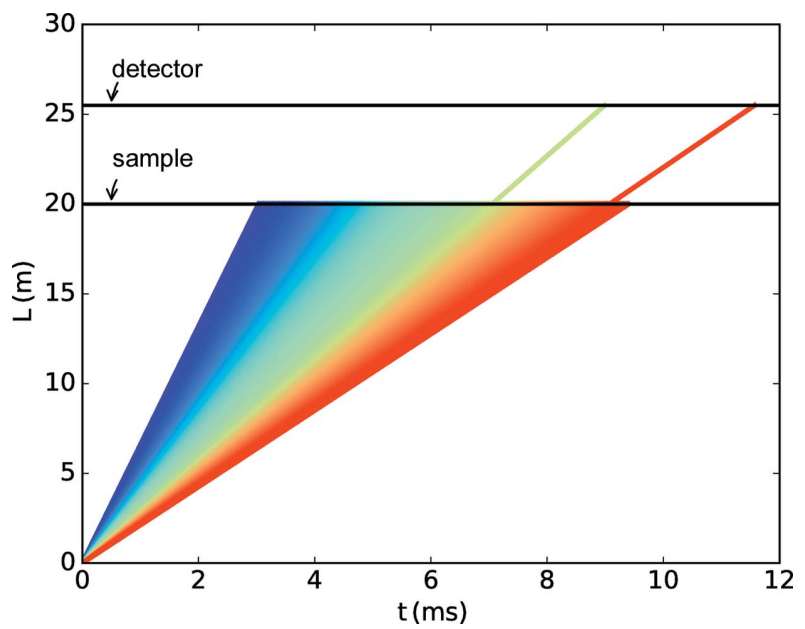

Figure 1

A timing diagram for a diffractometer with the sample position $20 \mathrm{~m}$ from the source and the detectors $25 \mathrm{~m}$ from the source. Note that the crystal at the sample selects some wavelengths and not others. Furthermore, there is a time difference between when neutrons of a given wavelength are at the sample and when they are at the detector.

energy physics community for some time (Ferbel, 1991; Nessi et al., 2003). However, the application is somewhat different in that the trigger is the detection event rather than a repetitive signal on a sample.

This article provides an overview of the event-based system at the SNS in $\S 2$, and gives a few examples of the diverse experiments in $\S 3$. Specifically, continuously ramping temperature measurements on POWGEN are discussed in $\S 3.1$, studies of battery degradation under cycling and phase transformations under heating or stress on VULCAN are discussed in $\$ 3.2$, studies of supercooled liquids on NOMAD are described in $\$ 3.3$, a study of the magnetic field phases of the multiferroic $\mathrm{MnWO}_{4}$ with a pulsed magnetic field on SEQUOIA are discussed in $\$ 3.4$, studies of the phonon density of states of a metallic glass over the glass transition on ARCS are discussed in $\S 3.5$, and a study of the phase stability of the Fermi chopper on SEQUOIA is provided in \$3.6. Other examples, such as rheological studies on the Liquids Reflectometer and continuous rotation measurements on the ARCS spectrometer, have also been performed and are detailed in their respective references (Adlmann et al., 2015; Weber et al., 2012).

Though in situ studies can be carried out at continuous sources (Isnard, 2007) to provide millisecond time scale acquisitions, such experiments use histogramming data acquisition. This work highlights the use of event-based acquisition and its ability to work at all time scales up to the speed of the instrument clock.

\section{Data structures and processing}

In this section the hardware for data collection and the necessary processing software for use at the SNS are described. Three types of data relevant to this paper are captured by the data-acquisition (DAQ) system: neutron event data, fast log data and slow log data. The accelerator timing system provides an absolute time stamp and a trigger signal correlated with the proton beam on the target $\left(T_{0}\right)$, the time when the neutrons are generated. The neutron event data and fast event data are time stamped in the data-acquisition hardware using time stamps, relative to a given $T_{0}$, with $100 \mathrm{~ns}$ resolution. Each detected neutron event is assigned a pixel identification (positional information) and a time stamp by the detector readout card, and is associated with the appropriate accelerator beam pulse.

For the fast event data, a custom analog-to-digital readout card (ADCROC), similar to the detector readout cards, digitizes and time stamps the signal. The ADCROC provides eight channels with 16-bit resolution over a scale of \pm 10 or $\pm 5 \mathrm{~V}$. In addition, there are 32 channels that can be used for digital input/output. These can be used to trigger external devices in time with the pulse on the target or to read a trigger pulse with the same $100 \mathrm{~ns}$ timing precision. A functional diagram of the ADCROC is provided in Fig. 2.

The card contains a field-programmable gate array (FPGA) for configuration and control of the fast logging. Aspects specific to different experiments will be described in the following sections. Several general configurations will be described here. The first is the ability to set a delay from $T_{0}$. This delay allows a fast signal to be triggered at the time when neutrons of a certain wavelength are passing the sample position. The second is that the sampling rate can be controlled by the level of the signal. This allows for efficient acquisition of data in experiments, like the pulsed-magnet experiment described in $\$ 3.4$, where there are long delays between the fast signals.

The neutron events and the data captured by the ADCROC are transported in the Adara data stream, with a digitized read back plus a time stamp for storage on disk (Shipman et al., 2014). The data-acquisition system aggregates the pulse information with the per pulse timing information to provide an absolute time stamp for each event with 100 ns resolution.

The slow log data are time stamped with an absolute time stamp, synchronized with a network time server to allow correlation with the neutron events and fast data logs.

All the data are saved as acquired in the event NeXus file (Könnecke et al., 2015). The NeXus format is a Pragma for a hierarchical data file (HDF 5) (HDF Group; http://www. hdfgroup.org/HDF5/) that allows multiple types of data to be saved together in an organized binary format. The fast and slow logs are saved under one entry in the data file. Therefore, the end user can work with all logs in the same manner. Neutron events are saved under a different entry in the file.

The data-acquisition software is built using the Experimental Physics and Industrial Control System (EPICS; EPICS Collaboration; http://www.aps.anl.gov/epics) tool kit. EPICS provides the interface to beamline equipment, including detectors, sample environment and experiment run control; network protocols (Channel Access and PVAccess) for transporting data and online visualization; and a rich suite of client tools. The graphical user interface and experiment automation are built using the EPICS Control System Studio 
toolkit (CSS Collaboration; http://controlsystemstudio.org). These tools provide a rich environment to quickly produce an integrated user interface for conducting the experiment.

With these event data in the NeXus file, processing can begin. To produce a data set suitable for analysis, the raw event data must be reduced with all the required corrections (e.g. vanadium normalization, empty sample environment etc.) and a binning regime and values chosen. Part of this procedure uses the event nature of the data to expedite processing (Peterson et al., 2015). The fact that the raw data remain untouched in event format means that they can be re-reduced at will, as many times as desired, with different parameters whenever needed, and either immediately or years later.

This contribution focuses on filtering and the use of those filtered events. Initially, one-off scripts were used to filter events. The thinking behind the development has now consolidated into flexible and efficient algorithms in the Mantid framework (Arnold et al., 2014). There are two main algorithms. GenerateEventsFilter determines where to filter the data on the basis of a $\log$, and FilterEvents filters the data on the basis of time stamps. The second algorithm will be described first. The goal is to filter data that have unique time stamps into bins (called workspaces) of identical conditions. This algorithm takes a three-column array, where the first column is the absolute time start, the second is the absolute time stop and the third is a bin indicator that allows equivalent data to be combined. Executing this algorithm puts data that occur between the start and stop time in the labeled workspace. That workspace is in a collection of workspaces so that the remainder of the reduction process can occur without explicit looping.

Filtering of events is usually the first step in a reduction chain but can occur anywhere before the data are histogrammed. Often, the filtering of events is used to correlate neutron events at the detector with an event that occurred at the sample position. Since the wavelength of the neutron is measured by time of flight, the timing diagram in Fig. 1 shows how the timing needs to be corrected for the distance between the sample and the detectors. The FilterEvents algorithm can correct for this timing difference in several ways. For the diffraction experiments it uses $L_{1} /\left(L_{1}+L_{2}\right)$, where $L_{1}$ is the source-to-sample distance and $L_{2}$ is the sample-to-detector distance. It also has the ability to use a custom array if that is needed. The aforementioned three-column array that is used for filtering is usually generated by the GenerateEventsFilter algorithm, but can be generated by hand or with a custom script if necessary.

The GenerateEventsFilter takes a $\log$ and generates the three-column workspace needed for FilterEvents. The user specifies the number and ranges for a $\log$ and then executes the algorithm. The output is then fed into the FilterEvents algorithm. Multiple outputs, obtained by running this algorithm with different logs, can be combined using logical operators. Then a single loop through the data will be used with the FilterEvents algorithm, making the process as efficient as possible. Execution efficiency is especially crucial when there are many events and many log values, as is the case for the stress example with VULCAN in $\$ 3.2$

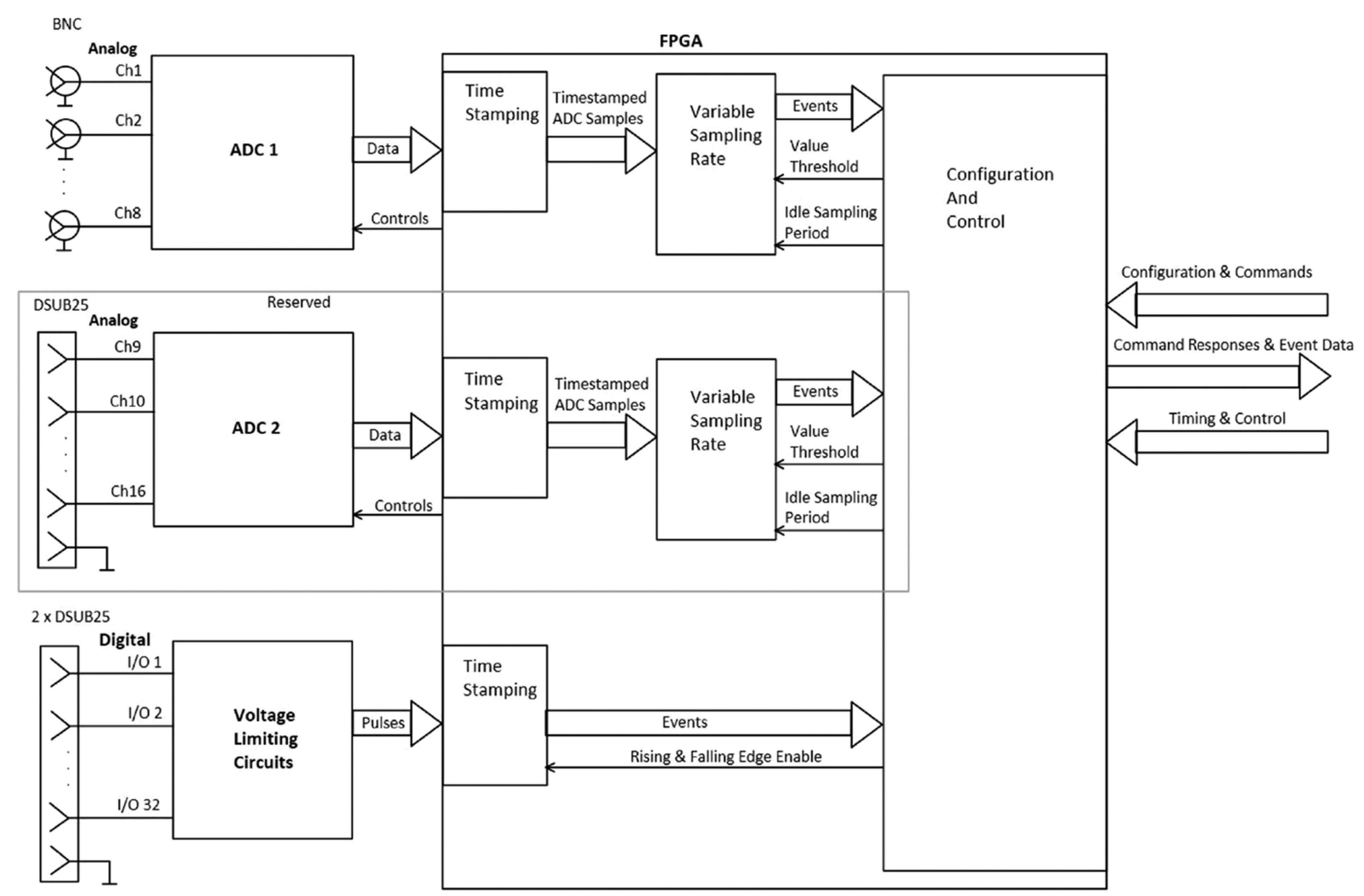

Figure 2

A block diagram for the ADCROC board for interfacing to fast sample environment equipment. 
These two algorithms are the most general for filtering events in Mantid. Specialized interfaces on top of these algorithms are built for powder diffraction reduction and in the PyVdrive (Zhou, 2017) tool for the VULCAN instrument.

\section{Experimental examples}

This section provides several examples from a wide variety of instrumentation. These examples are measurements where something is changing during the experiment. When designing such experiments one must be careful to understand the stability of the system over the time range of interest. This is handled in different ways for the different types of experiment. For continuously ramping temperature measurements, one ensures that the change in temperature is slow enough to be adiabatic. This may be accomplished by checking the temperature dependence in offline measurements, as in the spin glass example, or by adjusting the ramping rate to demonstrate that the physical properties are not changing more slowly than the ramp rate. For the load frame experiments, one instead wants to keep the oscillation above a certain rate to avoid unwanted creep or stress relaxation. Finally, in the pulsed-magnet case the researcher has to ensure that the temperature response due to the magnet pulse does not interfere with the magnetic response. The diversity of approaches for different experiments demonstrates that a careful understanding of the system is needed while designing the experiment.

\subsection{Continuously ramping temperature measurements of powder diffraction data on POWGEN}

One of the diffractometers at the SNS using event-based data reduction is the POWGEN high-resolution powder diffractometer (Huq et al., 2011). As a general-purpose powder diffractometer, it uses a variety of sample environments and cells. Most of this equipment provides some form of non-ambient conditions, be they low- or high-temperature gas flow or a specialized sample cell (e.g. electrochemical). Consequently, a range of different pieces of sample-related information can be entries in the metadata, including temperature, oxygen partial pressure, gas flow rates and cell voltage. More information is added as new sample environments are commissioned. POWGEN's $1 \AA$ bandwidth at $60 \mathrm{~Hz}$, coupled with the $2 \theta$ detector coverage at the time of its construction, limited the $d$-spacing range that could be covered in a single data set. During operation, bandwidth choppers were used to change the center wavelength of the $1 \AA$ band to select the $d$-spacing range of interest (Huq et al., 2011).

One way POWGEN uses the time stamps is to filter the data acquired while the temperature is continuously ramped into separate bins. The binning is based on the temperature log. This method is particularly useful for locating and studying phase transitions in materials. A material where this was tested was a polyhedral oligomeric silsesquioxane (POSS) known as octasilane-POSS $\left(\mathrm{C}_{16} \mathrm{H}_{56} \mathrm{O}_{20} \mathrm{Si}_{16}\right)$. POSS molecules are composed of large $\mathrm{Si}_{8} \mathrm{O}_{12}$ cages with a variety of possible ligands to tailor functionality. Different ligands can impart various properties, making them useful for catalysis, nanocomposites, biomaterials, molecular optics etc. (Cordes et al., 2010). A study was undertaken on POWGEN with a number of different POSS samples to provide high-quality structural data between 10 and $300 \mathrm{~K}$, supporting inelastic studies looking at motions of the ligands (Jalarvo et al., 2014). A $1.37 \mathrm{~g}$ sample of octasilane-POSS was one of the samples, posing a significant challenge for a time-of-flight neutron powder diffractometer as it contained 51.9 atom $\%$ hydrogen. The incoherent scattering from large atom fractions of hydrogen degrades data quality, making deuterated samples desirable for diffraction measurements in many cases. In the case of octasilane-POSS this was not feasible, but the sample was known to exhibit a phase transition at around $160 \mathrm{~K}$ that could be easily monitored using a very intense reflection located at a $d$ spacing of just under $11 \AA$. The sample was slowly cooled between 175 and $125 \mathrm{~K}$ in the POWGEN automatic sample changer at a rate of $0.2 \mathrm{~K} \mathrm{~min}^{-1}$. Fig. 3 shows contour plots that resulted from reducing the event data using different temperature bins between 12 and $1 \mathrm{~K}$. The different plots highlight the expected trade-off between temperature
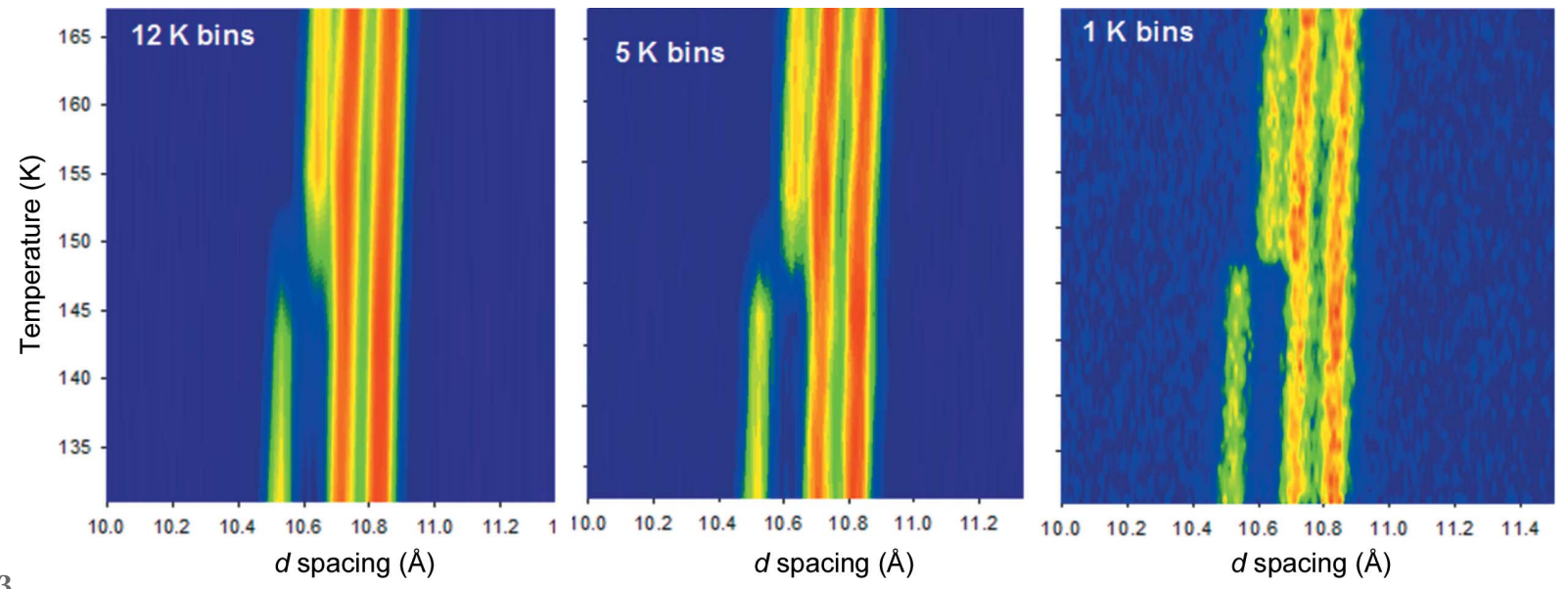

Figure 3

Contour plots of reduced data sets from octasilane-POSS $\left(\mathrm{C}_{16} \mathrm{H}_{56} \mathrm{O}_{20} \mathrm{Si}_{16}\right)$ across a phase transition, rebinned using different temperature intervals. 
resolution and noise. Although the $12 \mathrm{~K}$ temperature resolution had significantly less noise, the transition could be misidentified as a second-order phase transition with phase coexistence, whereas the $1 \mathrm{~K}$ resolution clearly reveals the classical sharp first-order behavior.

Rather than simply using the ability to re-reduce and slice existing data sets after the fact, the possibility also exists to exploit it actively. Such an approach could be seen as a timeefficient approach to map the phase behavior of a sample before collecting larger data sets at specific temperatures, should the process be irreversible or a second sample be unavailable. The major difficulty with using the slicing approach to map phase transitions routinely using a constant temperature ramp is that the accelerator at spallation sources occasionally trips, interrupting the neutron beam. If the beam did not recover quickly it would impact the statistics or leave a gap in the data. Should a gap coincide with the location of a phase transition this would seriously impact the usefulness of the data, possibly requiring a repeat experiment should

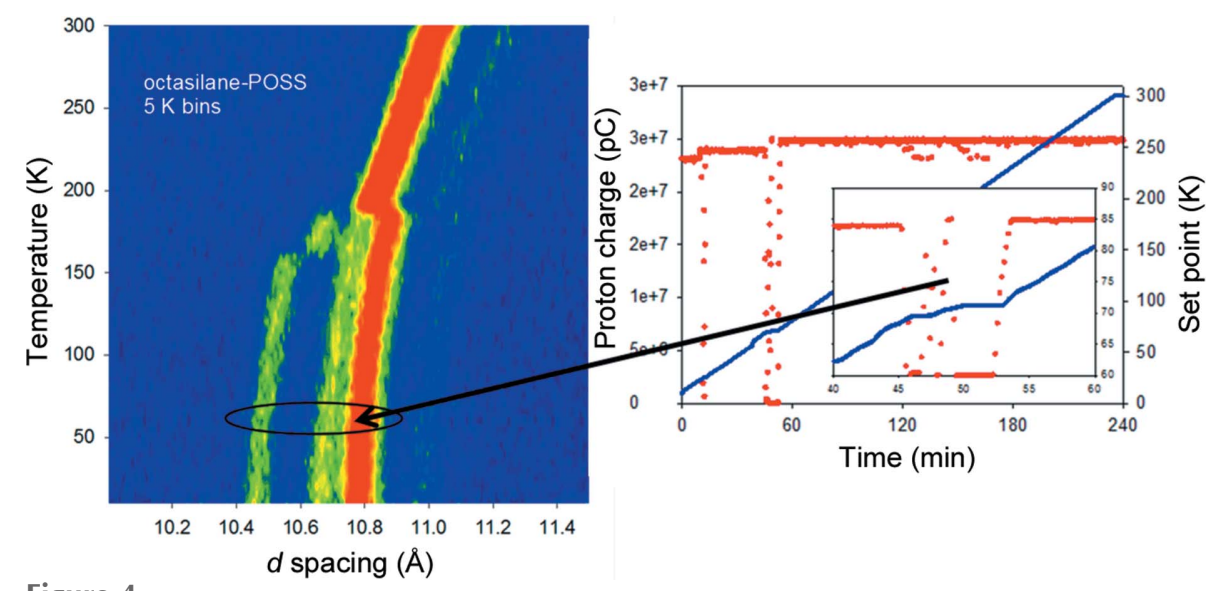

Figure 4

A contour plot of both transitions in octasilane-POSS during heating from 10 to $300 \mathrm{~K}$ at $2 \mathrm{~K} \mathrm{~min}^{-1}$ and the corresponding plot of temperature/proton charge versus time. The graph shows that the temperature holds when the beam has tripped. Had the temperature ramp not paused during this time there would be a gap in the data in the region indicated on the contour plot.
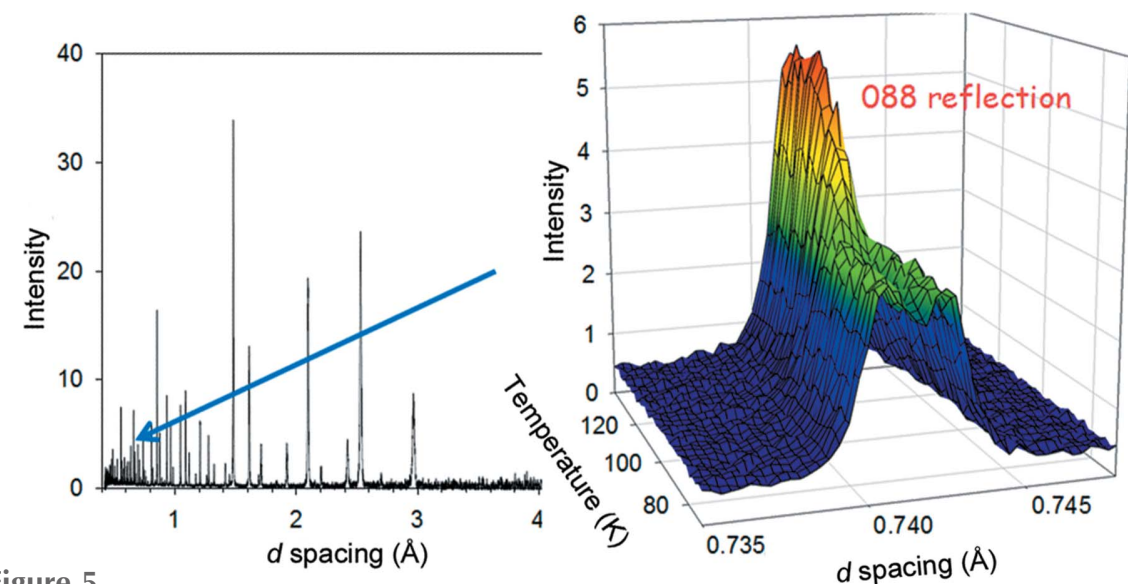

Three-dimensional plot of the 088 magnetite reflection over the Verwey transition in the range 90 $140 \mathrm{~K}$. The location of this reflection in the full data set is indicated, being outside the range usually accessible on a conventional laboratory powder diffractometer. sufficient sample remain. This problem is tackled in a straightforward manner. Diffraction data are collected continuously, and the ramp rate updated dynamically to maintain approximately constant counting statistics. The data can then be re-binned at will using the desired temperature interval with no gaps. To be more specific, data are usually collected at POWGEN in terms of the amount of proton charge on the target (in picocoulombs) rather than of time. This is the term that is used to normalize the data and it is a convenient method of monitoring the relative neutron flux on the sample. The ramp rate of the temperature controller is varied to maintain a constant $\delta_{\text {proton charge }} / \delta_{\text {temperature }}$. Using this metric, the temperature is held constant during a trip and ramp resumed, at a rate matching the returning beam when operation is restored. The EPICS-based control monitors the accelerator beam power. If the timepower drops below a threshold, the rate of change of available, the temperature will be held at a fixed value.

This mode of operation was first tested with a Lakeshore 340 cryocontroller using two samples during highpower accelerator testing in the POWGEN sample changer, which has a range of $10-300 \mathrm{~K}$. The beam power fluctuated frequently during this testing, allowing rigorous testing of the ratelimiting change in the control system.

The time available for testing was limited, so two samples were chosen that had previously exhibited very good signal-to-noise ratios in the region of interest. The first was the octasilanePOSS described above, which exhibits an extremely strong reflection near $11 \AA$ A, as previously seen in Fig. 3. On heating it exhibited two structural phase transitions between 120 and $200 \mathrm{~K}$ (as opposed to the single transition on cooling seen in Fig. 3), creating an opportunity to test the effectiveness of temperature slicing such data. Fig. 4 shows the diffraction data and temperature/proton charge versus time data for a data set collected on heating between 10 and $300 \mathrm{~K}$ at a nominal ramp rate of $2 \mathrm{~K} \mathrm{~min}^{-1}$ with a center wavelength of $3.731 \AA$ to capture the $11 \AA$ reflection. The diffraction data were sliced into $5 \mathrm{~K}$ bins in this instance. Too-coarse binning would be problematic in this case, as the two transitions were quite close together. The beam did trip for a period during the collection of this data set, showing the advantage of dynamic feedback in temperature ramping. Without the automatic 


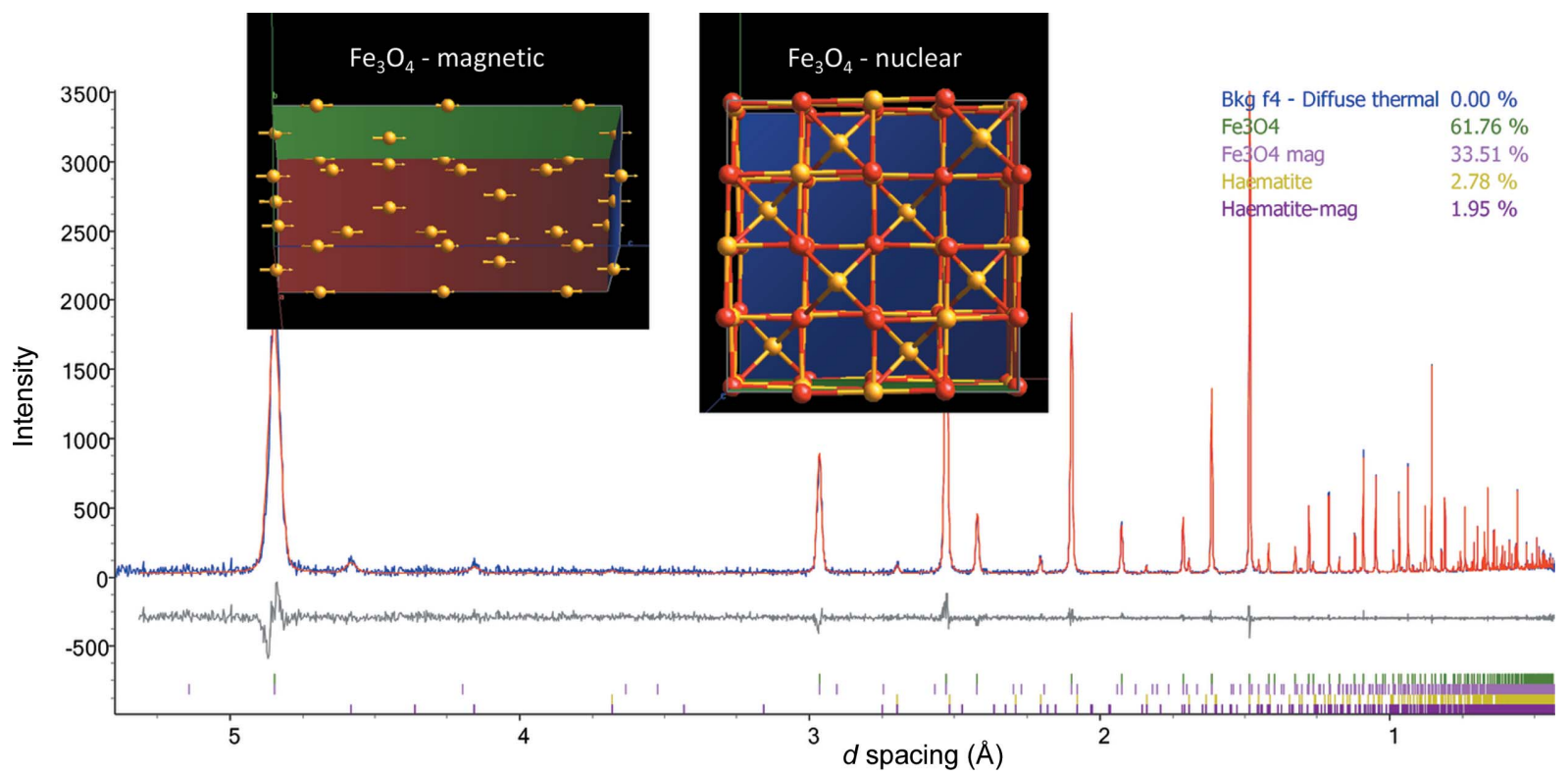

Figure 6

Refinement of the nuclear and ferrimagnetic structure of magnetite at $295 \mathrm{~K}$ from a $5 \mathrm{~K}$ slice (297.5-292.5 K). Hematite was present as a minor phase which exhibited a canted antiferromagnetic structure. This was nominally a 5 min data set, assuming a constant beam power.

temperature hold, a 'hole' or degraded statistics would have been apparent in the data between 50 and $55 \mathrm{~K}$.

The second sample was $2.36 \mathrm{~g}$ of magnetite $\left(\mathrm{Fe}_{3} \mathrm{O}_{4}\right)$ which exhibited the well known, if controversial, Verwey transition at $120 \mathrm{~K}$ (Verwey, 1939; Wright et al., 2001) and excellent statistics owing to the large sample mass. The data were collected on cooling between 300 and $10 \mathrm{~K}$ at a nominal rate of $1 \mathrm{~K} \mathrm{~min}^{-1}$ using a center wavelength of $1.333 \AA$. The Verwey transition was much more subtle than those of octasilanePOSS and was most easily visible in the $F d \overline{3} m 088$ reflection at $0.74 \AA$ A. Fig. 5 shows the data in this region, sliced every $2 \mathrm{~K}$.

Despite the reduced statistics from the fine temperature steps, the signal-to-noise level is more than sufficient to identify the start of the transition at $120 \mathrm{~K}$, exactly where it is expected. To demonstrate that a temperature-binned data set provided qualitative results, a full nuclear and magnetic refinement was carried out on data between 297.5 and $292.5 \mathrm{~K}$ (nominally $5 \mathrm{~min}$ at a rate of $1 \mathrm{~K} \mathrm{~min}^{-1}$ ), as shown in Fig. 6 .

Refinements were carried out using a beta version of TOPAS 6 (Coehlo, 2015, 2018). The magnetic structure of magnetite was described using the Shubnikov space group $166.101\left(R \overline{3} m^{\prime}\right)$. In order to fit the observed data, a minor hematite phase of about $2.81 \%$ needed to be included. It was necessary to include both its nuclear and magnetic peaks in the refinement. One intriguing aspect of the minor hematite phase is that it could not be described correctly in the conventional pure antiferromagnetic structure. Instead, the magnetic structure was set up in a basis-vector description using SARAh (Wills, 2000), with the resulting code inserted in the TOPAS input file. The final refinement yielded a canted antiferromagnetic structure for the hematite. A more detailed study of this aspect of the sample would need to be performed to verify this structure. Nevertheless, the fact that a few minutes of data are sufficient to demonstrate a deviation from the expected magnetic structure of a minority phase is essential, demonstrating the power of the POWGEN instrument and of the event-based acquisition technique.

\subsection{Event-based measurements of engineering materials on VULCAN}

Thanks to the high neutron flux at the engineering materials diffractometer VULCAN (Wang et al., 2010; An et al., 2011), processing of neutrons as events has been actively used for the continuous measurement of engineering, functional or energy materials under external monotonic or cyclic stimuli, e.g. thermal, mechanical and electrical fields. Event data acquisition provides advantages over stepwise measurements, for instance in a load frame test, where unwanted creep or stress relaxation may occur while the neutron counts accumulate during a specific step (An et al., 2011). Sample environment data, including dynamic mechanical signals, charger electrical signals, rapid AC voltage pulses, and induction heating temperature and power output etc., are recorded and time stamped as described in $\S 2$, and for fast logs, such as during fatigue measurements in the VULCAN load frame, data are recorded using the ADCROC. This unique event DAQ for both sample environments and neutrons allows a simple asynchronous pump-probe/stroboscopic approach to increase the time resolution into the microsecond regime, as demonstrated by the dynamic intensity response of piezoelectric materials under a $480.1 \mathrm{~Hz}$ AC field (An et al., 2012). The continuous and asynchronous measurements provide a simple and convenient experimental solution at the neutron instrument, where the user can actively concentrate on the planning and execution of experiments under external stimuli, while the sample environment and neutron data are post-processed and synchronized on the basis of the statistics of different time 
bins. Because of the aforementioned possible relaxation for the load frame experiment if a pause occurs during collection, the beam condition is handled differently than in the POWGEN continuously ramping temperature measurement case. Here, the beam status is monitored by the DAQ and users have an option to pause the load frame or not, depending on the concerns of material relaxation for loading segments (no relaxation occurs during unloading), but the system does not pause the collection of events coming from the ADCROC. The relative ease of implementation of the different cases of load frame and continuous temperature monitoring demonstrates the flexibility of the EPICS DAQ system.

Although the scattering intensity depends on the material and the scattering volume, often, for engineering materials measurements, sufficient statistics can be acquired in less than $1 \mathrm{~s}$. Therefore with the event mode of operation, neutron data can be binned to the sub-second regime. Combining this chopping with the stroboscopic methodology, VULCAN provides the capability for fast real-time operando testing. Users have benefited from these scientific capabilities and conducted a broad range of new research in materials science and engineering, including stress-induced twin reorientation (Pramanick et al., 2011), phase transformation in shapememory alloys (Yang et al., 2017; Wang et al., 2017) and bulk metallic glass composites (Wu et al., 2017), transformationinduced plasticity in alloys (Yu et al., 2014; Huang et al., 2017), twinning/detwinning behaviors in metals (Wu et al., 2013, 2014, 2015; Xie et al., 2017; Wu \& An, 2016), residual stress relaxation in engineering structures (Aba-Perea et al., 2016),
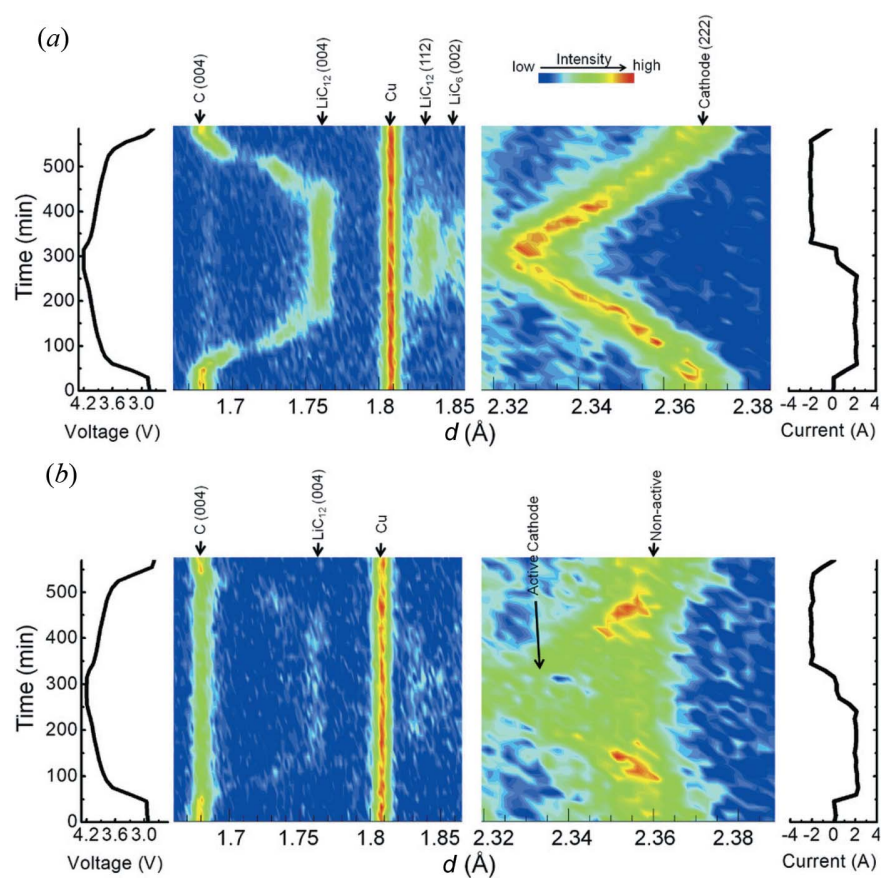

Figure 7

Contour plots during charge-discharge cycles, showing signs of degradation of a large-format battery $(a)$ before and $(b)$ after long-term cycling, with limited $\mathrm{Li}^{+}$insertion/removal processes in an active material (Cai, An et al., 2013). domain switching in oxides (Aman et al., 2014), non-equilibrium phase transformation during rapid heating (Yu et al., 2012), cycling and degradation of realistic-format batteries (Cai, An et al., 2013; Wang et al., 2012; Liu et al., 2013, 2016), and in situ materials processing and synthesis (Cai, Liu et al., 2013; Chen et al., 2015, 2014; Steiner et al., 2016). Specific examples of the use of event data acquisition are chosen from battery charge-discharge, rapid heating of a dual-phase steel and cyclic fatigue-induced martensitic phase transformation.

The first example is the study of the cathode and anode of a large-format battery. The idea is to use the diffraction pattern, as a function of time, to study the $\mathrm{Li}^{+}$insertion and removal process. Fig. 7 has false-color plots of the powder patterns, for everything in the battery, over a single charging cycle, $(a)$ for a pristine battery and $(b)$ after long-term cycling. Note that the Bragg-peak position provides information on the lattice and the intensity is indicative of the occupancy. Note also that the cathode Bragg peak 222, the lithiated anode peaks 002, 112 and 004, the 004 peak of the unlithiated anode, and a Cu Bragg peak from the leads are all visible.

The battery was positioned so that the beam traversed its middle. Other locations were checked as well for consistency. The battery was continuously charged and discharged by a

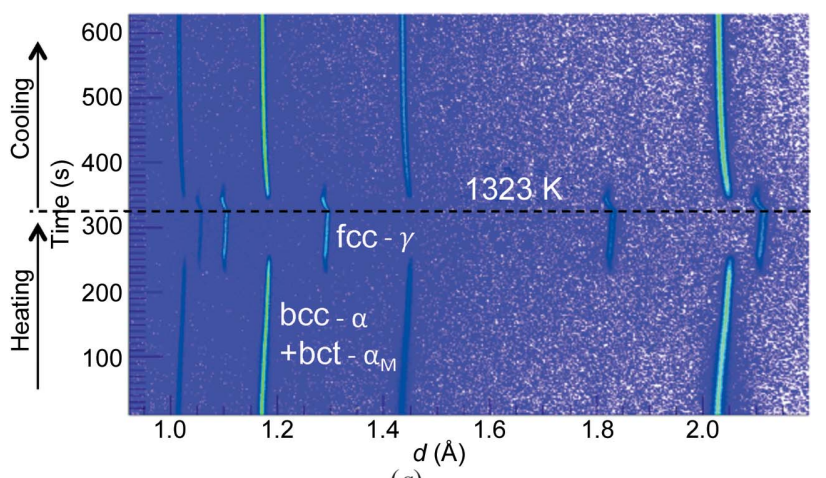

(a)

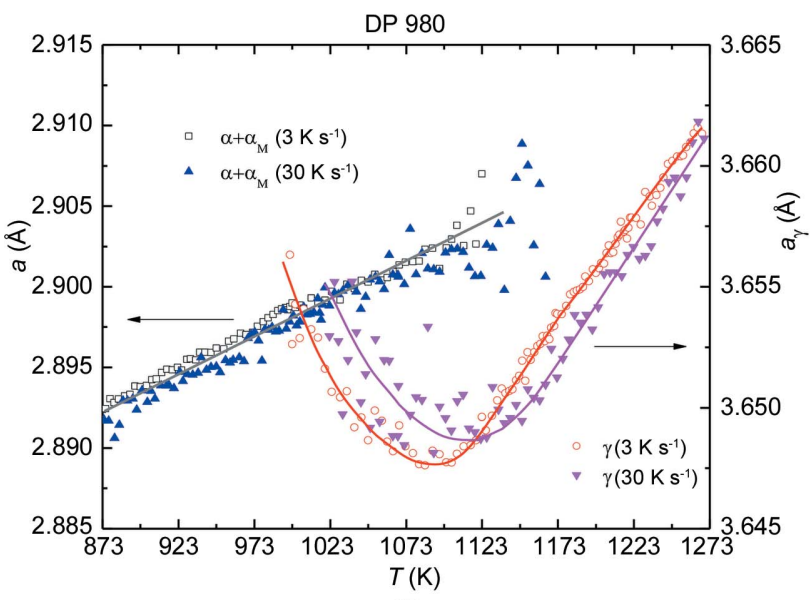

(b)

Figure 8

One second data binning $\left(3 \mathrm{~K} \mathrm{~s}^{-1}\right)$ and contour of a dual-phase steel during heating and cooling, revealing the phase transformation between ferrite $(\alpha)+$ martensite $\left(\alpha_{\mathrm{M}}\right)$ and austenite $(\gamma)$ phases, and the lattice behavior under higher heating rates as resolved by stroboscopic measurements (Yu et al., 2012). 
commercial charger, and the current and voltage were recorded by the ADCROC. Fig. 7 shows the results of one measured location. The dramatic change in the lattice position/time contour of the cathode 222 Bragg peak showed clear evidence of degradation as more $\mathrm{Li}^{+}$remains at the non-active lattice position after cycling. Furthermore, the graphite anode showed less lithiated compound after cycling. The state of charge of the healthy battery can be linked to the volume fraction of lithiated compounds or residual anode, and this quantitative information can be used to evaluate the inhomogeneity inside the large battery due to non-uniform degradation (Cai, An et al., 2013).

The next example is a study of phase transformation and heating/cooling rate for several as-received commercial steel samples. Fig. 8 shows the in situ measurement of a sample of a dual-phase steel used in the automobile industry. The sample is a $2 \times 8 \times 17 \mathrm{~mm}$ strip with a mass of $2.1 \mathrm{~g}$. The color contour plot shows the Bragg pattern for slow heating and cooling. It clearly shows the f.c.c.- $\gamma$ (f.c.c. is face-centered cubic) and b.c.c. $-\alpha+$ b.c.t. $-\alpha_{M}$ phases (b.c.c. is body-centered cubic and b.c.t. is body-centered tetragonal; the subscript $\mathrm{M}$ denotes martensitic). The bottom plot shows the lattice parameter change through the transition for both the faster cooling rate and the slower cooling rate.

The samples were rapidly heated using a pulsed DC current up to $300 \mathrm{~A}$. This setup provided a heating rate up to $30 \mathrm{~K} \mathrm{~s}^{-1}$. While a $1 \mathrm{~s}$ pattern from one single run can be reduced for $3 \mathrm{~K} \mathrm{~s}^{-1}$ heating, stroboscopic measurements were done at the fast heating rate of $30 \mathrm{~K} \mathrm{~s}^{-1}$. The post-synchronization of the data from the fast log of temperatures and neutron events was conducted on the basis of repeated measurements. As seen from the results, evidence for the influence of the heating rate on this specific alloy is provided by the delayed emergence and lattice change of the f.c.c. $-\gamma$ phase. A reduction in the f.c.c. lattice change during heating was also observed, which is thought to be associated with the concentration of $\mathrm{C}$ in the early formed f.c.c. $\gamma$ phase. Different phase transformation behavior was observed in other types of alloy measured by the same method (Yu et al., 2012).

The final example from VULCAN is to understand the cyclic fatigue behavior of 304 stainless steel. For this study, the load frame is oscillated at the desired frequency and the time stamp of the oscillation is recorded with the ADCROC. As mentioned earlier, in this case the load frame continued to cycle fast even during beam downtime, and was slowed slightly for selected cycles for continuous neutron measurement. Fig. 9 shows various plots illustrating the results.

A clear indicator of the martensite phase is the appearance of its 110 Bragg peak in Fig. 9(c). Nevertheless, other Bragg peaks of this phase are clearly visible over the extended $d$-spacing range shown in Fig. 9(b). While the phase transformation to the martensite phase usually occurs with relatively large deformation during monotonic loading, under cyclic loading it can occur under a small strain amplitude. Near about 200 cycles under $\pm 1 \%$ strain, detectable peaks characteristic of the martensitic phase are found in the diffraction contour, as shown in Fig. 9(c). The volume fraction of the detected martensitic phase shows a strong correlation with the
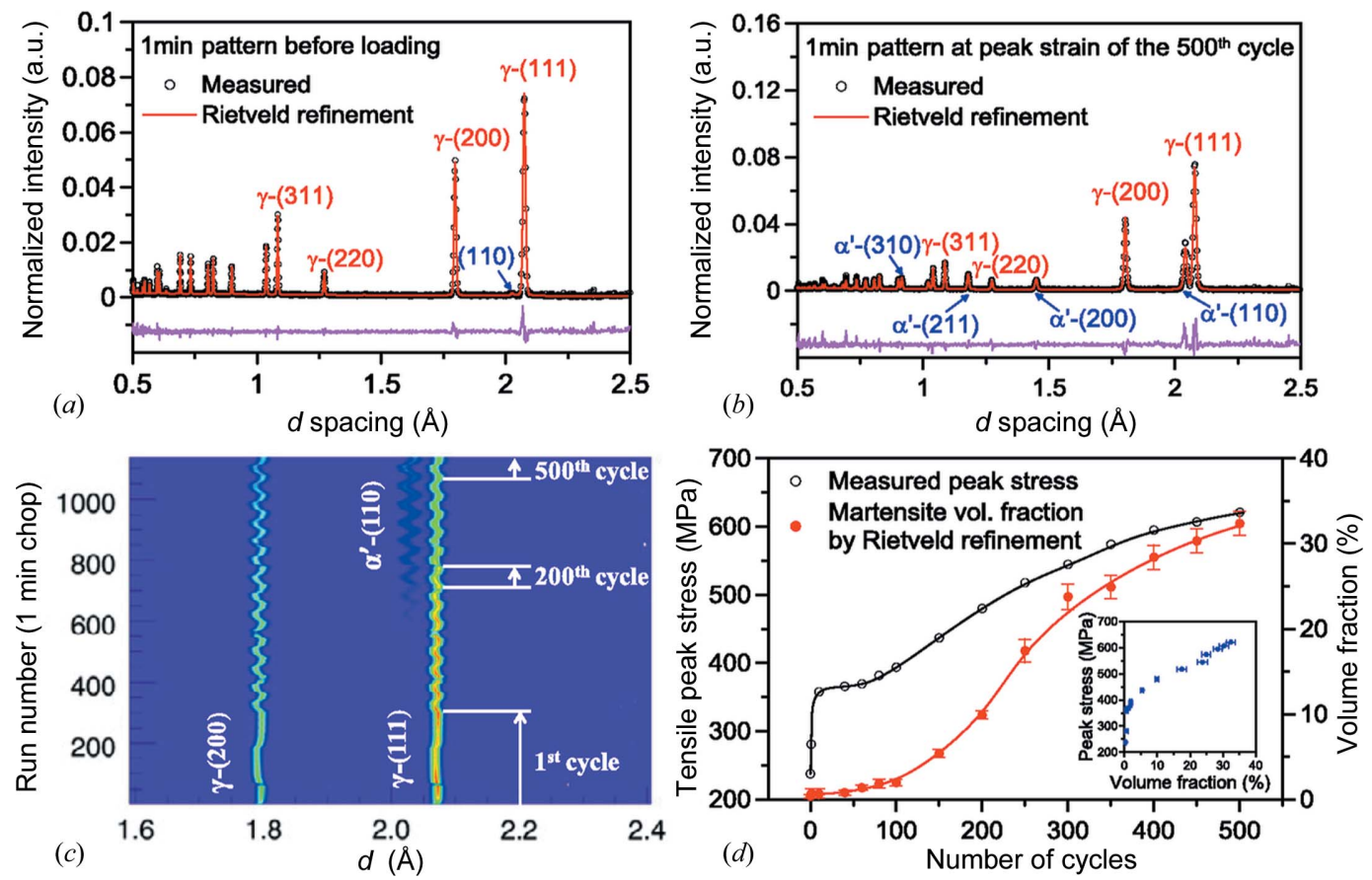

Figure 9

Neutron measurement of the stress-induced phase transformation in 304 stainless steel shows that the increase in the martensitic phase volume is responsible for the secondary hardening of the steel under cyclic loading. The powder diffraction patterns $(a)$ before loading and $(b)$ at the $1 \%$ peak strain of the 500th cycle are shown. (c) The $d$ spacing/time color contour plot, showing the evolution of the Bragg peaks with loading. (d) Evolution of the tensile peak stress and martensite phase volume fraction at the associated peak strain with a number of cycles. The inset in panel $(d)$ shows the correlation between the tensile peak stress and the martensite volume fraction (Yu et al., 2014). 
peak stress of the cycles, which indicates that secondary hardening is mainly due to the increase in the martensitic phase. The load sharing of the phases and the evolution of dislocations are also resolved by dynamic measurements ( $\mathrm{Yu}$ et al., 2014).

\subsection{Stroboscopic heating measurements on NOMAD}

The idea of stroboscopic data acquisition for neutron diffraction data (Eckold et al., 1998) has been used, albeit infrequently, for about 20 years. A process that is inherently too fast to be followed with sufficient statistics is repeated many times and the neutron data acquisition is subdivided into an appropriate number of channels, each representing a delay time relative to the start signal of the repeated process. The number of repetitions is given by the total time required to obtain sufficient statistics in each channel. This allows tracking of fast repeatable processes that are inaccessible in a 'singleshot' experiment. The process has to be repeatable over a timescale that allows for a sufficient number of neutron counts to be accumulated in each channel. Irreversible changes in the sample set an upper limit to this method. The shortest time scale that can be accessed by this method is limited by the precision with which a neutron can be attributed to a process time bin, and ultimately by the residence time of the neutron in the sample; it is of the order of microseconds. Event-based data acquisition allows for a very elegant implementation of stroboscopic data acquisition, in that the density of the time bins can be changed after the fact and adapted to the counting statistics needed in each bin.

We have used event-based stroboscopic data acquisition on the NOMAD diffractometer (Neuefeind et al., 2012) to study supercooled high-temperature melts. Supercooled liquids are transient structures and are thermodynamically unstable relative to the crystal. A higher cooling rate decreases the

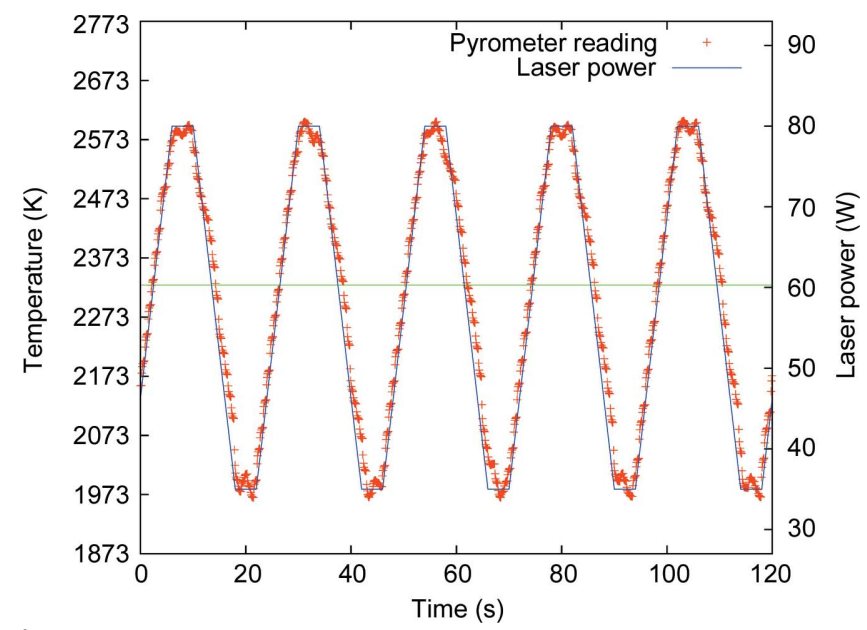

Figure 10

A section of the temperature cycle of supercooled $\mathrm{Al}_{2} \mathrm{O}_{3}$ in the aerodynamic levitator. The red symbols represent the pyrometrically measured temperature while the solid blue line is the laser output power related to the scale on the right-hand side. The melting point of $\mathrm{Al}_{2} \mathrm{O}_{3}$ is $2327 \mathrm{~K}$ (Paradis et al., 2004) and is indicated as a horizontal line. No crystallization of $\mathrm{Al}_{2} \mathrm{O}_{3}$ takes place. The total measuring time was $1 \mathrm{~h}$. probability of crystallization. The specific sample chosen for this study was a spheroid of $\mathrm{Al}_{2} \mathrm{O}_{3}$, with a mass of $\sim 30 \mathrm{mg}$ and $3 \mathrm{~mm}$ in diameter. It was held in an aerodynamic levitator that minimizes contact with other materials that can act as heterogeneous nucleation sites. Laser heating of the sample is used and a pyrometer provides contactless temperature measurements (Weber et al., 2014). The laser power can be cycled at a controlled rate. With these components, a stable and reproducible temperature cycle can be established between the stable and the supercooled liquid, as demonstrated in Fig. 10. Specifically, the latent heat involved in the crystallization process would mean the temperature, as measured by the pyrometer and indicated by the red points, would deviate significantly from the laser power, shown by the blue line. Since the temperature is continuously streamed with the event data, neutrons can then be correlated with the state of the sample.

The structure factor curves $S(Q)$ for two example temperatures are shown in Fig. 11. These two curves were generated by filtering the events acquired continuously during the temperature ramps shown in Fig. 10. Note that no Bragg peaks, indicative of crystalline order, are present in either curve, demonstrating that the sample is indeed liquid over the entire temperature range and into the supercooled region. Furthermore, the results presented here are consistent with previous measurements of $\mathrm{Al}_{2} \mathrm{O}_{3}$ (Landron et al., 2001).

\subsection{Diffraction in high magnetic fields with pulsed magnets}

As an example that uses fast experiment logs, we summarize event filtering of a diffraction measurement with a pulsed magnetic field. The pulsed magnet is of the minicoil design of H. Nojiri that is in use at a broad range of facilities (Yoshii et al., 2009; van der Linden et al., 2008; Islam et al., 2009; Noe et al., 2013). The magnet produces a truncated sine wavefield

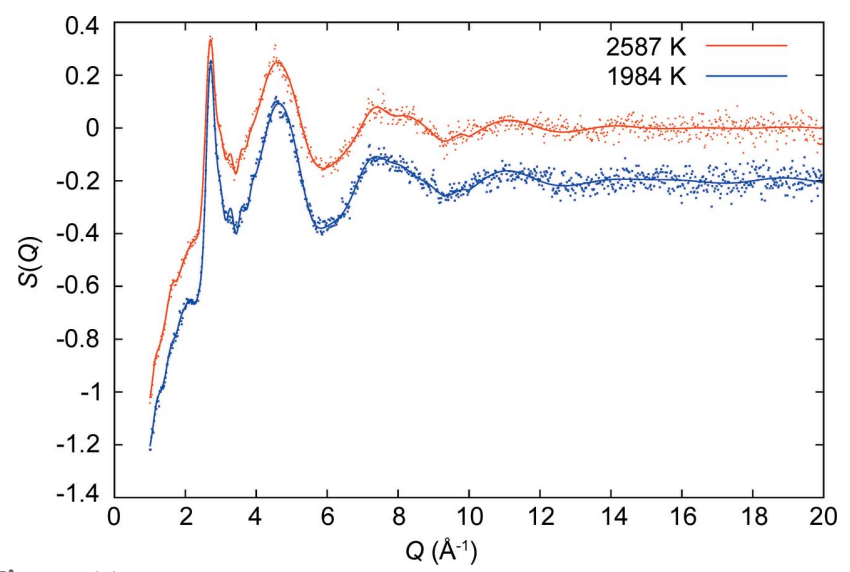

Figure 11

Structure factors for an $\mathrm{Al}_{2} \mathrm{O}_{3}$ spheroid at the highest $(2587 \mathrm{~K}$, red symbols and line) and lowest temperature (1984 K, blue symbols and line). The absence of Bragg reflections at either temperature demonstrates that no crystallization takes place. The solid line is a Fourier filtered version of the symbols. The low-temperature curve is displaced by 0.2 units. The principal diffraction peak at about $2.7 \AA^{-1}$ sharpens and moves to a slightly higher $Q$, commensurate with higher density and higher ordering at lower temperature. 
pulse of up to $H=32$ T with an $\sim 2.6 \mathrm{~ms}$ FWHM. An example pulse is shown in Fig. 12.

The magnet sits in an automatically filled bath of liquid nitrogen for cooling. The magnet can be fired repeatedly at a frequency limited by the cooling rate of the magnet after a pulse. The maximum repetition rate at $32 \mathrm{~T}$ is about $7 \mathrm{~min}$. The pulsed magnet has been utilized both on the SEQUOIA spectrometer (Granroth et al., 2006, 2010), set up in diffraction mode by removing the Fermi chopper, and on the CORELLI diffractometer (Rosenkranz \& Osborn, 2008). Initial experiments were carried out before the ADCROC, described in $\S 2$, was developed. In this case, the field pulses were recorded on a storage scope, using the same trigger as the magnet, and the time and field were correlated after the experiment. Currently, the field pulse is recorded in the same event file as the data and can be used for live data monitoring. Here is a brief description of the setup. The ADCROC is configured through the EPICS interface to output a TTL (transistor-transistor logic) trigger pulse at a rate determined by the necessary cool-down time of the magnet. Usually, the pulse is then delayed to an offset away from the $T_{0}$ cycle to correlate the time when neutrons of the desired wavelength are passing the sample with the time when the peak field is on the sample. The TTL pulse also triggers an oscilloscope to record the field pulse so it can be compared with a predetermined shape. If it does not match, a digital input port that allows the ADCROC to provide the trigger pulse is latched low until the measured pulse shape can be investigated by personnel. The same signal that is recorded by the scope is sent to an analog input of the ADCROC for recording with the neutron data.

An obvious way to set up an experiment would be to time the peak field so that it matches the time of flight for neutrons of the desired wavelength as they traverse the sample. However, as the wavelength is encoded with the time of flight, the pulse can be phased so multiple field-dependent peaks are observed. An example of this technique is demonstrated in $\mathrm{MnWO}_{4}$. This system has a high-field phase where an incom-

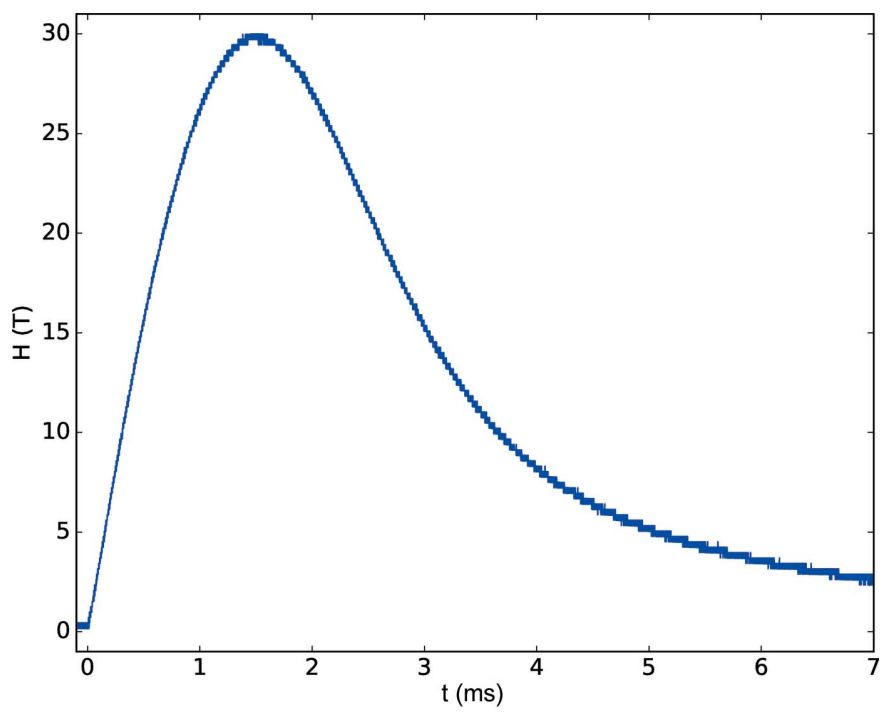

Figure 12

An example of a field pulse generated by the pulsed magnet. mensurate magnetic peak is located at the $\left(\frac{1}{4} \frac{1}{2} \frac{1}{2}\right)$ position and another phase, at a lower field, with the incommensurate peak at (-0.214 $\frac{1}{2}$ 0.457) (Nojiri et al., 2011). By tuning the timing of the field pulse, both the high- and low-field peaks can be observed without the need to perform independent data collections. Such an efficiency gain is crucial, since the cooldown time of the magnet means only 150 field pulses are combined to provide the observed statistics. This observation is illustrated in Fig. 13. In the lower panel, the points show the counts in two different detectors (one in blue and the other in red) as a function of wavelength. The top panel shows the field as neutrons of that wavelength traverse the sample. The peak in the high-field phase (blue) is not observed in the low-field phase, and conversely the peak in the low-field phase (red) is not observed in the high-field phase. Nevertheless, they are both observed in a single pulse when the wavelength and field as a function of time are coincident. This technique has been used to map out fully the high-field phases in $\mathrm{LiNiPO}_{4}$ (ToftPetersen et al., 2017).

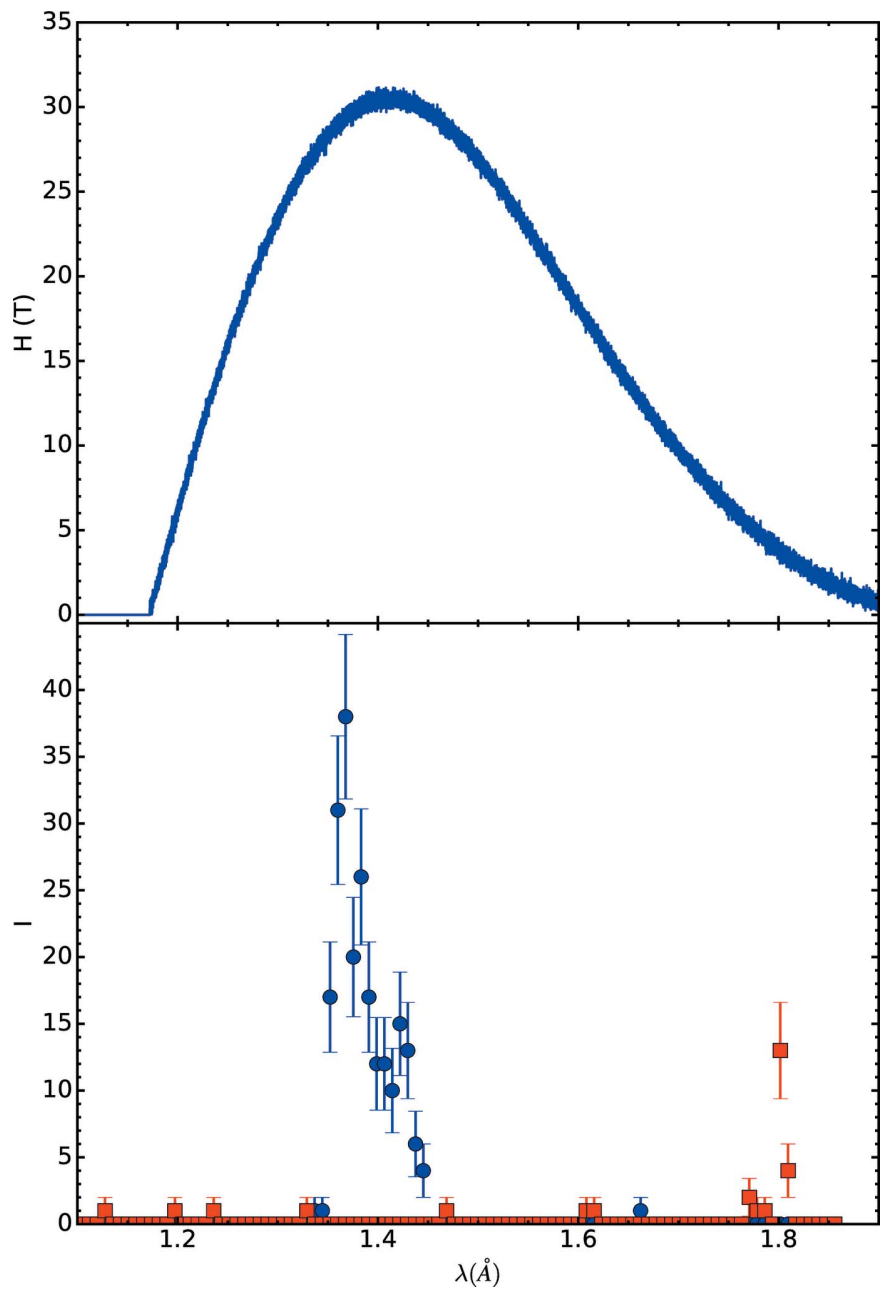

Figure 13

(Top) A field pulse. (Bottom) The Bragg peaks observed when the field is on in two different detectors, one indicated by blue points and the other by red points. Time of flight has been converted to wavelength to show the correlation between field and wavelength that allows the observation of Bragg peaks in two specific phases during the same field pulse. 
To produce the results in Fig. 13, the data were reduced using the event-filtering algorithms in the following manner. First the data are filtered so that the time during nitrogen fills is removed. Then the data are filtered into field-on and fieldoff pulses. The field-on pulses are further filtered into pulses on the sample of a set field range. Typically, this binning is 2 or $5 \mathrm{~T}$ wide. For the zero-field measurements additional filtering is performed. Just after a field pulse, the temperature of the sample can rise by as much as $10 \mathrm{~K}$, assuming the cryostat is operating at a base temperature of $4 \mathrm{~K}$. The data during this time are not useful, so a second filtering of the field-off pulses is conducted on the basis of temperature to remove the neutrons during the temperature excursion. Therefore, this example also provides a clear demonstration of using the reduction routines in a successive manner, as mentioned in $\$ 2$.

\subsection{Temperature-dependent phonon densities of states through the glass transition in metallic glasses}

The Wide-Angular-Range Chopper Spectrometer (ARCS) is often used for studies of temperature-dependent phonon scattering for powders and single crystals (Abernathy et al., 2012). Typically, data are acquired in $30-40 \mathrm{~min}$ scans at multiple temperature set points, and data are not collected while the sample environment is heating, cooling or equilibrating to a new temperature. However, as described in $\$ 3.1$, an obvious use of event data is to make use of the sample environment information in the logs to bin the data by temperature. For example, when acquiring long scans at a single temperature to obtain a phonon density of states, this approach could be used to remove data at the beginning of a scan before the sample temperature has equilibrated. However, the high neutron flux available at ARCS also allows for more sophisticated use of temperature binning to access new scientific information not available with histogrammed data.

The ARCS instrument has been used to acquire information about the changes in vibrational entropy while metallic glasses undergo their glass transition from an amorphous solid to an undercooled liquid (Smith et al., 2017). Neutron scattering measurements on glasses are routinely performed at ambient temperature (Suck et al., 1980). However, studies of metallic glasses above the glass transition, in the undercooled liquid state, are limited by the rapidly intervening crystallization of the liquid. Fig. 14(a) shows the heat-capacity curve of the metallic glass $\mathrm{Cu}_{46} \mathrm{Zr}_{46} \mathrm{Al}_{46}$ during continuous heating at $2 \mathrm{~K} \mathrm{~min}^{-1}$ obtained with differential scanning calorimetry (DSC). The heat capacity shows a characteristic increase in heat flow at the onset of the glass transition at $687 \mathrm{~K}$, at which point the amorphous solid becomes an undercooled liquid. This is followed shortly by the sharp peak of crystallization at $732 \mathrm{~K}$. The $\sim 20 \mathrm{~min}$ between 687 and $732 \mathrm{~K}$ is the critical time frame of interest when the glass is an undercooled liquid.

The ability to assess this brief time window with inelastic neutron scattering relied on a high and stable neutron flux, and the ability to post-process the data flexibly in this time window. Once the material has crystallized, the experiment

cannot easily be repeated because formation of the glass requires rapid cooling of the order of $1000 \mathrm{~K} \mathrm{~s}^{-1}$ (Johnson et al., 2011). During the neutron experiment, the metallic glass was continuously heated at $2 \mathrm{~K} \mathrm{~min}^{-1}$ while the neutron data were acquired. Following the experiment, the data are binned in $\Delta 2 \mathrm{~K}$ increments and the elastic scattering is extracted and plotted as a function of temperature. This reveals the temperature at which the onset of crystallization occurs during the neutron experiment. This temperature is known precisely from DSC experiments performed at the same heating rate. Thermal lag and temperature offset between the thermocouple and the sample were addressed in the neutron experiments by performing an off-line test using a thermocouple embedded into the sample and operating the furnace under identical heating conditions. This revealed a constant thermal lag of $6 \mathrm{~K}$ over the temperature range of interest for the experiment.

The phonon density of states (DOS) is extracted by summing data with a temperature change of 4,10 or $25 \mathrm{~K}$, with the energy axis binned every 1,2 or $3 \mathrm{meV}$. For example, a DOS obtained at $402 \mathrm{~K}$ with $\Delta 4 \mathrm{~K}$ is obtained by summing data collected between 400 and $404 \mathrm{~K}$, which have an average temperature of $402 \mathrm{~K}$. Post-binning of the data with multiple

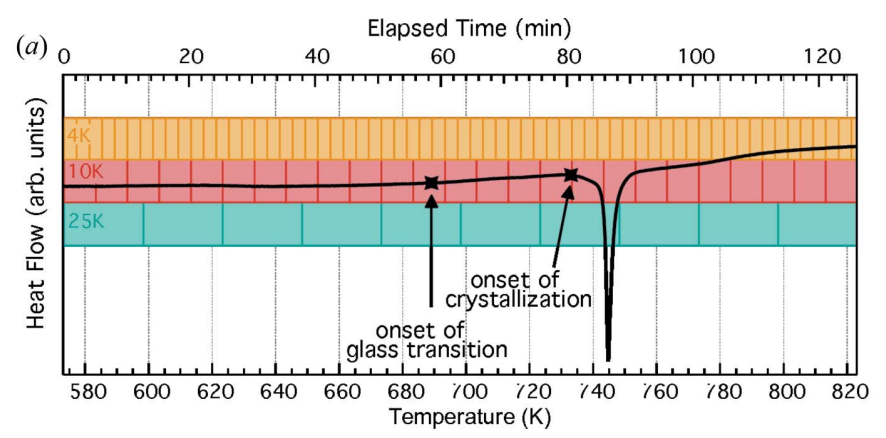

(b)

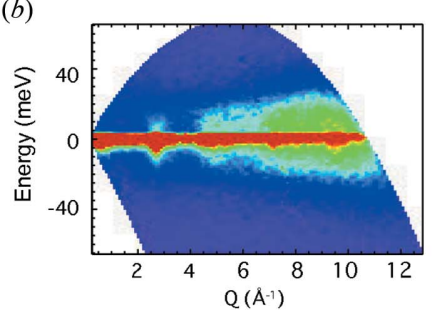

(d)

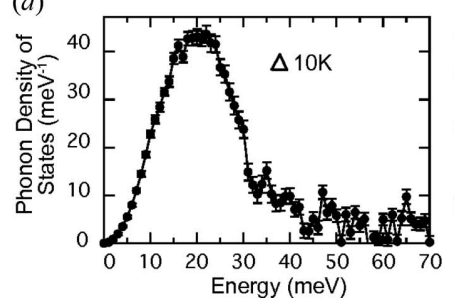

Figure 14

The study of the vibrational entropy of the metallic glass $\mathrm{Cu}_{46} \mathrm{Zr}_{46} \mathrm{Al}_{46}$ through the narrow time window of its glass transition is made possible by the ability to optimize the number and statistical quality of spectra obtained by binning data in temperature every 4,10 and $25 \mathrm{~K}$ and in energy between 1 and $3 \mathrm{meV}$. (a) DSC identifies the region of interest between 687 and $732 \mathrm{~K}$. (b) The $S(Q, E)$ and $(c)$ the phonon DOS at $665 \mathrm{~K}$ from $\triangle 4 \mathrm{~K}$ of data. $(d),(e)$ The phonon DOSs at $664 \mathrm{~K}$ from $\Delta 10 \mathrm{~K}$ of data and at $662 \mathrm{~K}$ from $\Delta 25 \mathrm{~K}$ of data, respectively. 
temperature and energy binnings is necessary to ensure that sufficient statistical quality is achieved to extract quantitative information from the spectra. Furthermore, and like the diffraction study of POSS in Fig. 3, it is necessary to ensure that a subtle change in the phonon DOS and vibrational entropy is not overlooked by binning over too large a range in temperature. Fig. 14(b) shows an $S(Q, E)$ plot obtained from data at $665 \mathrm{~K}$ with $\Delta 4 \mathrm{~K}$. This represents $\sim 0.1$ coulombs of proton charge on target (C) acquired in $2 \mathrm{~min}$, and shows reasonable statistical quality for obtaining a phonon DOS for an energy binning of $2 \mathrm{meV}$, as shown in Fig. 14(c). The remaining Figs. 14(c)-14(e) provide a comparison of trading off temperature and energy bin size to obtain the physical answer required by the study. Data at $664 \mathrm{~K}$ for $\Delta 10 \mathrm{~K}$, or $\sim 0.2 \mathrm{C}$ of data acquired in $5 \mathrm{~min}$, and data at $662 \mathrm{~K}$ for $\Delta 25 \mathrm{~K}$, or $\sim 0.6 \mathrm{C}$ of data acquired in $12 \mathrm{~min}$, result in the phonon DOSs shown in Figs. 14(d) and 14(e), respectively. The largest $\Delta T$ in Fig. 14(e) gives the smallest error bars and the most consistency with the least amount of noise at higher energies. The smallest $\Delta T$ in Fig. 14(c) gives the largest error bars and tends to smoother features of the DOS at lower energies owing to the larger energy bin size. The data from $\Delta 10 \mathrm{~K}$ in Fig. 14(d) give the best compromise to extract values for vibrational entropy from the DOS.

This experiment, to extract the vibrational entropy from the phonon DOS as a metallic glass traverses its glass transition, demonstrates the importance of high neutron flux and the ability to post-process data in multiple ways. The narrow $\sim 20$ min time window of interest cannot be satisfactorily assessed when the data are histogrammed during collection, as this does not allow for a critical recalibration of temperature and a highly detailed analysis to ensure subtle changes in vibrational entropy are not overlooked. Therefore, eventbased acquisition and processing are critical to access the phonon DOS of the glass in its undercooled liquid state.

\subsection{Testing chopper timing with event data}

Another useful feature of event-based acquisition is that it can be used to test the performance of instrument components, like the stability of chopper phasing. For the first five years of operation, the SNS accelerator timing followed a smoothed version of the line frequency at a nominal $60 \mathrm{~Hz}$ (Henderson et al., 2014). The Fermi choppers of the ARCS and SEQUOIA spectrometers were designed to follow this phase variation. Nevertheless, it is important to identify how closely the choppers match the specification. In 2012, a reevaluation of the accelerator power supplies led to some tests of operating the accelerator at a fixed $60 \mathrm{~Hz}$. Fig. 15 shows the change of phase from one pulse to the next, before, during and after the fixed $60 \mathrm{~Hz}$ frequency operation. Note that, quite frequently, the phase variation is more than half a cycle change. These tests were successful and now the accelerator is operated at a fixed $60 \mathrm{~Hz}$. These accelerator tests provided the opportunity to see the degree to which a more stable input signal allowed the chopper to follow the input signal more closely. They also provided the opportunity to study how a rather large change in phase stability affects the performance of the chopper.

These tests were performed on the SEQUOIA spectrometer. This is a fine-resolution direct-geometry spectrometer that uses a Fermi chopper to monochromate the beam (Granroth et al., 2006, 2010). In the tightest chopping configuration, the full width of the neutron pulse through the chopper may be $10 \mu$ s. Therefore, any timing variation can change the neutronics performance. The phase of a given chopper is measured by recording its top dead center (TDC) signal in the event stream. The TDC signal is generated each time the chopper makes a full revolution. In the event stream, in a similar manner to the ADCROC, the first two bytes of the event record are used to differentiate a TDC signal from a neutron event.

For these specific tests, 840021 neutron pulses were analyzed when the accelerator was following the line frequency. For comparison, 359895 pulses were analyzed at a fixed frequency of $60 \mathrm{~Hz}$. While following the line frequency, the accelerator phase was limited to change with a maximum slew rate of $1 \mathrm{mHz} \mathrm{s}^{-1}$. Fermi chopper No. 2 was phased for $100 \mathrm{meV}$ at a rotation speed of $600 \mathrm{~Hz}$. All the data were acquired in a single run and then filtered into histograms, one where the accelerator followed the $60 \mathrm{~Hz}$ line frequency and the other where it was fixed at $60 \mathrm{~Hz}$. The histogram bins are the $100 \mathrm{~ns}$ ticks of the clock. The histograms are plotted in Fig. 16, where the red points are the fixed $60 \mathrm{~Hz}$ and the blue points are where the accelerator followed the line frequency. The solid lines are fits of the data to Gaussian distributions. For the line-frequency-following case, the peak amplitude is centered at $(-1.4 \pm 1.4) \times 10^{-2} \mu \mathrm{s}$, and the width is $(6.3 \pm$ $0.7) \times 10^{-2} \mu$ s. The center is shifted slightly to $(1.08 \pm 0.01) \times$ $10^{-2} \mu$ s and the width of $(1.4734 \pm 0.0009) \times 10^{-1} \mu$ s is broader when the accelerator is following the line frequency. Also, when following the line frequency there are instances of TDC signals as far as $50 \mu$ s away from the center, whereas for the fixed frequency the maximum excursion is $3 \mu$ s. The

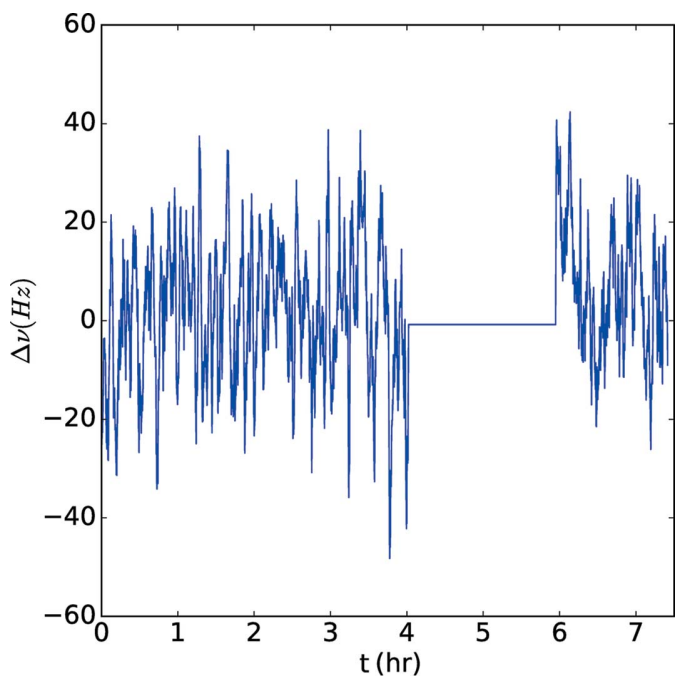

Figure 15

The change in phase from one pulse to the next, before, during and after the fixed $60 \mathrm{~Hz}$ test. 


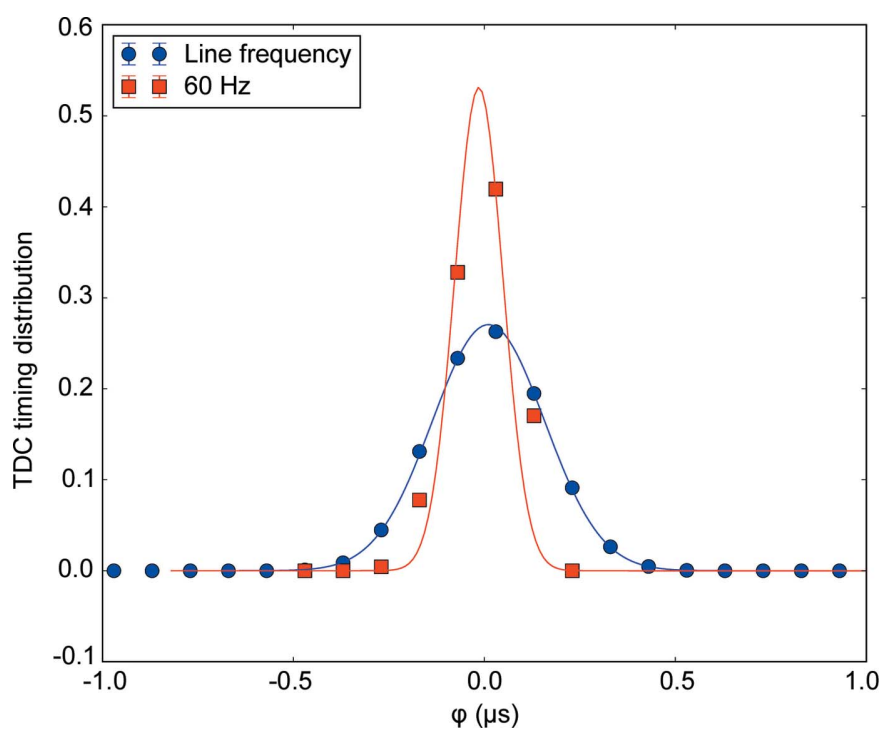

Figure 16

The distribution of chopper TDC signals when the signal is at a fixed $60 \mathrm{~Hz}$ (red squares) and when it follows the line frequency (blue circles).

Gaussian shape does not fully capture the wings of the fixedfrequency case. Nevertheless, the width shows that the distribution narrows as the accelerator distribution tightens. Regardless, for both cases the width of the distribution is less than the $1 \mu$ s phase uncertainty requirement to observe no effect on the data.

\section{Conclusions}

Event-based data acquisition and analysis have enabled novel experiments on the instruments at the SNS. These experiments have been carried out on the diffractometers and spectrometers with varying parameters like temperature, strain, magnetic field and charge. Furthermore, they have covered a broad range of systems, including batteries, steels, bulk metallic glasses, magnetic systems, metal-organics and many more. The use of this technique is only expected to expand in the future.

\section{Acknowledgements}

We are grateful to Matt Rucker, Bekki Mills and Lou Santodonato for technical support with sample environments in the experiments described here. For the pulsed-magnet experiment, we have benefited from a long-term collaboration with the group of H. Nojiri at Tohoku University, Japan. The authors thank Dr Mike Crawford (Dupont) and Dr Emil Bozin (Brookhaven National Laboratory) for permission to use their octasilane-POSS and magnetite samples, respectively, during development of the dynamic ramping methodology. The accelerator team including John Galambos were helpful with the fixed-frequency accelerator study.

\section{Funding information}

HLS acknowledges support from the US Department of Energy, Office of Basic Energy Sciences, under contract No.
DE-FG02-03ER46055. Early demonstrations of the ADCROC DAQ and asynchronous measurements on VULCAN, and the development of the pulsed-magnet environment into a user capability, were supported by separate Laboratory Directed Research Development projects at Oak Ridge National Laboratory. Neutron scattering measurements are sponsored by the User Facilities Division, Office of Basic Energy Sciences, US Department of Energy. This manuscript has been authored by UT-Battelle, LLC, under contract No. DE-AC05-00OR22725 with the US Department of Energy.

\section{References}

Aba-Perea, P., Pirling, T. \& Preuss, M. (2016). Mater. Des. 110, $925-$ 931.

Abernathy, D. L., Stone, M. B., Loguillo, M. J., Lucas, M. S., Delaire, O., Tang, X., Lin, J. Y. Y. \& Fultz, B. (2012). Rev. Sci. Instrum. 83, 015114.

Adlmann, F. A., Gutfreund, P., Ankner, J. F., Browning, J. F., Parizzi, A., Vacaliuc, B., Halbert, C. E., Rich, J. P., Dennison, A. J. C. \& Wolff, M. (2015). J. Appl. Cryst. 48, 220-226.

Aman, A., Chen, Y., Lugovy, M., Orlovskaya, N., Reece, M. J., Ma, D., Stoica, A. D. \& An, K. (2014). J. Appl. Phys. 116, 013503.

An, K., Riedel, R. A., Miller, S. D., Kohl, J. A., Choo, H. \& Jones, J. (2012). Asynchronous In Situ Neutron Scattering Measurement of $<10 \mu$ s Transient Phenomena at Spallation Neutron Source. Oak Ridge National Laboratory, Oak Ridge, Tennessee, USA.

An, K., Skorpenske, H. D., Stoica, A. D., Ma, D., Wang, X.-L. \& Cakmak, E. (2011). Metall. Mater. Trans. A, 42, 95-99.

Arnold, O. et al. (2014). Nucl. Instrum. Methods Phys. Res. A, 764, 156-166.

Cai, L., An, K., Feng, Z., Liang, C. \& Harris, S. J. (2013). J. Power Sources, 236, 163-168.

Cai, L., Liu, Z., An, K. \& Liang, C. (2013). J. Mater. Chem. A, 1, 6908 6914.

Chen, Y., Rangasamy, E., dela Cruz, C. R., Liang, C. \& An, K. (2015). J. Mater. Chem. A, 3, 22868-22876.

Chen, Y., Yang, L., Ren, F. \& An, K. (2014). Sci. Rep. 4, 5179.

Coehlo, A. A. (2015). TOPAS 6 Beta. Coelho Software, Brisbane, Australia.

Coelho, A. A. (2018). J. Appl. Cryst. 51, 210-218.

Cordes, D. B., Lickiss, P. D. \& Rataboul, F. (2010). Chem. Rev. 110, 2081-2173.

Dalgliesh, R. M., Lau, Y. G. J., Richardson, R. M. \& Riley, D. J. (2004). Rev. Sci. Instrum. 75, 2955-2959.

Eckold, G., Hagen, M. \& Steigenberger, U. (1998). Phase Transitions, 67, 219-244.

Ferbel, T. (1991). Experimental Techniques in High-Energy Nuclear and Particle Physics. Singapore: World Scientific.

Granroth, G. E., Kolesnikov, A. I., Sherline, T. E., Clancy, J. P., Ross, K. A., Ruff, J. P. C., Gaulin, B. D. \& Nagler, S. E. (2010). J. Phys. Conf. Ser. 251, 012058.

Granroth, G. E., Vandergriff, D. H. \& Nagler, S. E. (2006). Physica B, 385-386, 1104-1106.

Henderson, S. et al. (2014). Nucl. Instrum. Methods Phys. Res. A, 763, 610-673.

Huang, H., Wu, Y., He, J., Wang, H., Liu, X., An, K., Wu, W. \& Lu, Z. (2017). Adv. Mater. 29, 1701678.

Huq, A., Hodges, J. P., Gourdon, O. \& Heroux, L. (2011). Z. Kristallogr. Proc. 1, 127-135.

Islam, Z., Ruff, J. P. C., Nojiri, H., Matsuda, Y. H., Ross, K. A., Gaulin, B. D., Qu, Z. \& Lang, J. C. (2009). Rev. Sci. Instrum. 80, 113902.

Isnard, O. (2007). C. R. Phys. 8, 789-805.

Jalarvo, N., Gourdon, O., Ehlers, G., Tyagi, M., Kumar, S. K., Dobbs, K. D., Smalley, R. J., Guise, W. E., Ramirez-Cuesta, A., Wildgruber, C. \& Crawford, M. K. (2014). J. Phys. Chem. C, 118, 5579-5592. 
Johnson, W. L., Kaltenboeck, G., Demetriou, M. D., Schramm, J. P., Liu, X., Samwer, K., Kim, C. P. \& Hofmann, D. C. (2011). Science, 332, 828-833.

Könnecke, M. et al. (2015). J. Appl. Cryst. 48, 301-305.

Landron, C., Hennet, L., Jenkins, T. E., Greaves, G. N., Coutures, J. P. \& Soper, A. K. (2001). Phys. Rev. Lett. 86, 4839-4842.

Linden, P. J. E. M. van der, Mathon, O., Strohm, C. \& Sikora, M. (2008). Rev. Sci. Instrum. 79, 075104.

Liu, H., Chen, Y., Hy, S., An, K., Venkatachalam, S., Qian, D., Zhang, M. \& Meng, Y. S. (2016). Adv. Energ. Mater. 6, 1502143.

Liu, H., Fell, C. R., An, K., Cai, L. \& Meng, Y. S. (2013). J. Power Sources, 240, 772-778.

Mason, T. E. et al. (2006). Physica B, 385-386, 955-960.

Nakatani, T., Inamura, Y., Moriyama, K., Ito, T., Muto, S. \& Otomo, T. (2014). JPS Conf. Proc. 1, 014010.

Nessi, M., Nordberg, M., Jenni, P. \& Smith, K. (2003). ATLAS HighLevel Trigger, Data Acquisition and Controls: Technical Design Report. ATLAS-TDR-016. CERN, Geneva, Switzerland.

Neuefeind, J., Feygenson, M., Carruth, J., Hoffmann, R. \& Chipley, K. K. (2012). Nucl. Instrum. Methods Phys. Res. B, 287, 68-75.

Noe, G. T. II, Nojiri, H., Lee, J., Woods, G. L., Léotin, J. \& Kono, J. (2013). Rev. Sci. Instrum. 84, 123906.

Nojiri, H., Yoshii, S., Yasui, M., Okada, K., Matsuda, M., Jung, J.-S., Kimura, T., Santodonato, L., Granroth, G. E., Ross, K. A., Carlo, J. P. \& Gaulin, B. D. (2011). Phys. Rev. Lett. 106, 237202.

Paradis, P.-F., Ishikawa, T., Saita, Y. \& Yoda, S. (2004). Jpn. J. Appl. Phys. 43(4R), 1496.

Peterson, P. F., Campbell, S. I., Reuter, M. A., Taylor, R. J. \& Zikovsky, J. (2015). Nucl. Instrum. Methods Phys. Res. A, 803, 2428.

Pramanick, A., An, K., Stoica, A. D. \& Wang, X.-L. (2011). Scr. Mater. 65, 540-543.

Rosenkranz, S. \& Osborn, R. (2008). Pramana J. Phys. 71, 705-711.

Satoh, S., Muto, S., Kaneko, N., Uchida, T., Tanaka, M., Yasu, Y., Nakayoshi, K., Inoue, E., Sendai, H., Nakatani, T. \& Otomo, T. (2009). Nucl. Instrum. Methods Phys. Res. A, 600, 103-106.

Shipman, G., Campbell, S., Dillow, D., Doucet, M., Kohl, J., Granroth, G., Miller, R., Stansberry, D., Proffen, T. \& Taylor, R. (2014). 2014 IEEE 10th International Conference on e-Science, Vol. 1, pp. $223-$ 230. Piscataway: IEEE.

Smith, H. L., Li, C. W., Hoff, A., Garrett, G. R., Kim, D. S., Yang, F. C., Lucas, M. S., Swan-Wood, T., Lin, J. Y., Stone, M. B., Abernathy, D. L., Demetriou, M. D. \& Fultz, B. (2017). Nat. Phys. 13, 900-905.

Steigenberger, U., Eckold, G. \& Hagen, M. (1994). Nucl. Instrum. Methods Phys. Res. B, 93, 316-321.

Steigenberger, U., Eckold, G. \& Hagen, M. (1995). Physica B, 213214, 1012-1016.

Steiner, M. A., Calhoun, C. A., Klein, R. W., An, K., Garlea, E. \& Agnew, S. R. (2016). J. Nucl. Mater. 477, 149-156.

Suck, J. B., Rudin, H., Guntherodt, H. J., Beck, H., Daubert, H. \& Glaser, W. (1980). J. Phys. C. Solid State Phys. 13, L167-L172.
Toft-Petersen, R., Fogh, E., Kihara, T., Jensen, J., Fritsch, K., Lee, J., Granroth, G. E., Stone, M. B., Vaknin, D., Nojiri, H. \& Christensen, N. B. (2017). Phys. Rev. B, 95, 064421.

Verwey, E. J. W. (1939). Nature, 144, 327-328.

Wang, D., Mu, J., Chen, Y., Qi, Y., Wu, W., Wang, Y., Xu, H., Zhang, H. \& An, K. (2017). J. Alloys Compd. 696, 1096-1104.

Wang, X.-L., An, K., Cai, L., Feng, Z., Nagler, S. E., Daniel, C., Rhodes, K. J., Stoica, A. D., Skorpenske, H. D., Liang, C., Zhang, W., Kim, J., Qi, Y. \& Harris, S. J. (2012). Sci. Rep. $2,747$.

Wang, X.-L., Holden, T. M., Stoica, A. D., An, K., Skorpenske, H. D., Jones, A. B., Rennich, G. Q. \& Iverson, E. B. (2010). First Results from the VULCAN Diffractometer at the SNS. Materials Science Forum, Vol. 652, Mechanical Stress Evaluation by Neutrons and Synchrotron Radiation, edited by Y. Akiniwa, K. Akita \& H. Suzuki, pp. 105-110. Zürich: Trans Tech Publications.

Weber, F., Rosenkranz, S., Pintschovius, L., Castellan, J.-P., Osborn, R., Reichardt, W., Heid, R., Bohnen, K.-P., Goremychkin, E. A., Kreyssig, A., Hradil, K. \& Abernathy, D. L. (2012). Phys. Rev. Lett. 109, 057001.

Weber, J. K. R., Benmore, C. J., Skinner, L. B., Neuefeind, J., Tumber, S. K., Jennings, G., Santodonato, L. J., Jin, D., Du, J. \& Parise, J. B. (2014). J. Non-Cryst. Solids, 383, 49-51.

Wills, A. S. (2000). Physica B, 276-278, 680-681.

Windsor, C. G. (1981). Pulsed Neutron Scattering. London: Taylor and Francis

Wright, J. P., Attfield, J. P. \& Radaelli, P. G. (2001). Phys. Rev. Lett. 87, 266401.

Wu, W. \& An, K. (2016). J. Alloys Compd. 656, 539-550.

Wu, W., An, K., Huang, L., Lee, S. Y. \& Liaw, P. K. (2013). Scr. Mater. 69, 358-361.

Wu, W., Liaw, P. K. \& An, K. (2015). Acta Mater. 85, 343-353.

Wu, W., Qiao, H., An, K., Guo, X., Wu, P. \& Liaw, P. K. (2014). Int. J. Plast. 62, 105-120.

Wu, Y., Ma, D., Li, Q. K., Stoica, A. D., Song, W. L., Wang, H., Liu, X. J., Stoica, G. M., Wang, G. Y., An, K., Wang, X. L., Li, M. \& Lu, Z. P. (2017). Acta Mater. 124, 478-488.

Xie, Q., Liang, J., Stoica, A. D., Li, R., Yang, P., Zhao, Z., Wang, J., Lan, H., Li, R. \& An, K. (2017). Scr. Mater. 137, 83-87.

Yang, H., Yu, D., Chen, Y., Mu, J., Wang, Y. D. \& An, K. (2017). Mater. Sci. Eng. A, 680, 324-328.

Yoshii, S., Ohoyama, K., Kurosawa, K., Nojiri, H., Matsuda, M., Frings, P., Duc, F., Vignolle, B., Rikken, G. L. J. A., Regnault, L.-P., Michimura, S. \& Iga, F. (2009). Phys. Rev. Lett. 103, 077203.

Yu, D., An, K., Chen, Y. \& Chen, X. (2014). Scr. Mater. 89, 45-48.

Yu, Z., Feng, Z., An, K., Zhang, W., Specht, E. D., Chen, J., Wang, X.-I. \& David, S. (2012). Trends in Welding Research, Proceedings of the 9th International Conference, June 4-8, 2012, Chicago, Illinois, USA. Materials Park: ASM International.

Zhou, W. (2017). PyVdrive - A Data Reduction and Analysis Software for (SNS) VULCAN Based on PyQt. https://github.com/neutrons/ PyVDrive. 